\title{
Optimizing astrophotonic spatial reformatters using simulated on-sky performance
}

\author{
Theodoros Anagnos ${ }^{\mathrm{a}, \mathrm{e}}$, Robert J. Harris ${ }^{\mathrm{a}}$, Mark K. Corrigan ${ }^{\mathrm{b}}$, Andrew P. Reeves ${ }^{\mathrm{c}}$, Matthew J. \\ Townson $^{\text {b }}$, David G. MacLachlan ${ }^{\mathrm{d}}$, Robert R. Thomson ${ }^{\mathrm{d}}$, Tim J. Morris ${ }^{\mathrm{b}}$, Christian Schwab,f, \\ and Andreas Quirrenbach ${ }^{\mathrm{a}}$ \\ ${ }^{a}$ Landessternwarte, Zentrum für Astronomie der Universität Heidelberg, Königstuhl 12, 69117 \\ Heidelberg, Germany \\ ${ }^{b}$ Centre for Advanced Instrumentation, Durham University, South Road, Durham DH1 3LE, \\ UK \\ ${ }^{c}$ Deutsches Zentrum für Luft-und Raumfahrt (DLR), Oberpfaffenhofen, 82234 Weßling, \\ Germany \\ ${ }^{\mathrm{d} S U P A}$ (Scottish Universities Physics Alliance), Institute of Photonics and Quantum Sciences, \\ Heriot-Watt University, Edinburgh, EH14 4AS, UK \\ e Department of Physics and Astronomy, Macquarie University, NSW 2109, Australia \\ ${ }^{\mathrm{f}}$ The Australian Astronomical Observatory (AAO), Level 1, 105 Delhi Rd, North Ryde, NSW, \\ 2113, Australia
}

\begin{abstract}
One of the most useful techniques in astronomical instrumentation is image slicing. It enables a spectrograph to have a more compact angular slit, whilst retaining throughput and increasing resolving power. Astrophotonic components like the photonic lanterns and photonic reformatters can be used to replace bulk optics used so far. This study investigates the performance of such devices using end-to-end simulations to approximate realistic onsky conditions. It investigates existing components, tries to optimize their performance and aims to understand better how best to design instruments to maximize their performance. This work complements the recent work in the field and provides an estimation for the performance of the new components.
\end{abstract}

Keywords: instrumentation, adaptive optics, spectrographs, photonic lantern, astrophotonics, image slicing, simulations

\section{INTRODUCTION}

Over last decade one of the major goals driving the development of astronomical instrumentation has been the detection of an Earth-like planet orbiting either a Sun-like star or an M-dwarf. Whilst this is challenging, the science driver is also very exciting; the exploration of small possibly-habitable exoplanets inside the Goldilocks zone (e.g., Ref. 1,2). One of the most successful methods to date is the radial velocity technique, where the presence of a planet is inferred through movements in the stellar lines. To use the radial velocity method to detect these small planets, sub-m/s radial velocity precision in the measurements is needed. This requires a highly stable spectrograph that has been carefully calibrated.

Harnessing the temporal stability of the single-mode (SM) fiber's spatial profile output (close to the diffraction limit) as the spectrograph's input, is one of the solutions to making this task easier (e.g., Ref. 3-6). However, the atmosphere limits the coherence of the light, forming a seeing disk instead of a diffraction limited point spread function (PSF) resulting in considerable coupling losses. As a consequence, most current astronomical telescopes operate in the seeing limited regime - the multi-mode (MM) regime of photonics, where coupling of starlight is easier than in the SM regime. However, the required size of the spectrograph gets larger in the seeing limited

Further author information: (Send correspondence to Th.A.)

Th.A.: E-mail: tanagnos@lsw.uni-heidelberg.de, Telephone: (+49) 06221-54-1727 
regime, demanding large fragile optics in order to maintain high spectral resolving power $(\mathrm{R}>100,000)$ on large telescopes. $^{7}$

In the seeing limited regime, the size of a dispersive spectrograph is dependent on the diameter of the telescope. $^{8}$ This dependence imposes a correlation of the number of the spatial modes forming the telescope's PSF being analogous to the ratio of square of the telescope's diameter $D_{\mathrm{T}}$ over the Fried seeing parameter $\mathrm{r}_{0} \cdot{ }^{9-11}$

Currently the largest ground-based telescopes are 8-10 $\mathrm{m}$ in diameter requiring large spectrographs, to couple the light efficiently while also achieving high resolving power (e.g., Ref. 12-14). This problem will be even more challenging for spectrograph designers when the Extremely Large Telescopes (ELT, GMT, TMT) are built. ${ }^{15-17}$

A solution for this is to use adaptive optics (AO) systems to reduce the number of spatial modes. Some extreme AO systems manage to get close to the diffraction limited regime ( $>90 \%$ Strehl ratio) in the H-band, but these demand a very bright guide star to operate properly (e.g., Ref. 18-21). Their performance also degrades at visible wavelengths, limiting the available science targets.

Another approach, with less severe constraints on image quality is to reduce the size of the instrument by spatial reformatting. By using this technique, the PSF is reformatted into a different geometry. This is conceptually similar to image slicing (Ref. 22, and references therein). The sampled PSF can be divided in smaller pieces that can be coupled to smaller and more stable instruments. ${ }^{9,23,24}$ It should be noted, however, that most implementations of reformatting do not retain spatial information and therefore are not suitable for scientific goals requiring spatially resolved information.

Harnessing photonic technologies to implement this concept has led to a variety of new devices, to name a few the PIMMS (Photonic Integrated Multimode Micro Spectrograph), ${ }^{25}$ the photonic dicer (PD) ${ }^{26}$ the hybrid reformatter $(\mathrm{HR})^{11}$ and the Photonic TIGER device. ${ }^{27}$ The majority of these devices are derived from the photonic lantern (PL), ${ }^{28-30}$ and consist of different optical fiber/inscribed waveguides geometries. The PL is a device that bridges the MM and the SM regimes by having a MM core to the one end and many SM cores at the other end. Initially, PLs were constructed with fibers (e.g., Ref. 30,31), but later were manufactured using other methods

(e.g., Ref. 10,32). They allow the PSF to be coupled into instruments more efficiently than SM fibers. ${ }^{33,34}$

A major benefit of operating in the SM regime is the absence of modal noise in spectroscopic measurements, enabling better calibration of the acquired measurements than before. ${ }^{35}$ Coupling the time changing MM input to the spectrograph creates the modal noise, which induces movement of the barycenter for any given wavelength. As a result, more noise is added into the spectroscopic measurements reducing the precision (e.g., Ref. 36-41). Conversely, by using a SM fiber the modal noise is eliminated, as only the fundamental mode propagates (not counting different polarization states), while the higher order modes radiate out into the cladding of the fiber.

Over the last decade, astrophotonic reformatters have been used to combine the high coupling efficiency of a MM fiber and the noise elimination properties of a SM fiber. However, special care should be given to the instrument configuration for sources of modal noise at early stages of the optical set-up. ${ }^{42,43}$ Furthermore, there is no spatial preservation of the coupled image into astrophotonic reformatters in comparison to a conventional image slicer. Nevertheless, this is not a limitation for high resolution spectroscopy, as these devices are often placed after a MM fiber and additional components such as scramblers and modal noise elimination devices, producing a stable PSF (Ref. 2), but not preserving the spatial information.

In this paper, we analyze the simulated results of an astrophotonic reformatter, namely the $\mathrm{HR},{ }^{11}$ and compare it with its on-sky performance. This is an astrophotonic spatial reformatter that geometrically manipulates the MM input PSF into a SM (diffraction-limited) pseudo-slit output aiming to increase the resolving power of the spectrograph into which the light is coupled, and enable more precise measurements of astronomical targets with the elimination of modal noise. The HR is composed of a 92-core multicore fiber (91 plus an extra core to define orientation and aid alignment) tapered down to form a $\mathrm{PL}^{28-30}$ having a MM entrance at one end face, while towards the other end the cores are uncoupled in the original hexagonal arrangement of the multicore fiber. Following that, the cores are connected to a ultrafast laser inscription (ULI) reformatter that re-arranges the hexagonal geometry of the multicore fiber cores into a slit profile output to feed a spectrograph.

This paper is organized as follows: starting with Section 2 the configuration parameters for the simulation of the HR are reported. In Section 3 results are presented as well as the selected method and the optimization 
Table 1. Soapy input parameters for simulation

\begin{tabular}{lccc}
\hline \hline & \multicolumn{3}{c}{ Modes of AO operation } \\
\hline & closed-loop & open-loop & tip-tilt \\
\hline \hline Parameters & & & \\
\hline Seeing (arcsec) & 0.69 & 0.69 & 0.69 \\
Instantaneous Strehl ratio (mean) & 0.53 & 0.21 & 0.11 \\
Long exposure Strehl ratio (mean) & 0.38 & 0.04 & 0.06 \\
Fried parameter $r_{0}$ (m) (@1550 nm) & 0.15 & 0.15 & 0.15 \\
Atmosphere layers & 5 & 5 & 5 \\
DM integrator loop gain tip-tilt & 0.3 & 0.001 & 0.3 \\
DM integrator loop gain Piezo & 0.3 & 0.001 & 0.001 \\
\hline \hline
\end{tabular}

techniques. Following that, a discussion of the results is included in Section 4, and finally we conclude in Section 5 .

\section{METHODS}

Performing precise simulations is of paramount importance for calibration of future designs as well as to estimate their performance in realistic conditions. Motivated by this, we used a combination of two simulation tools to simulate an astrophotonic reformatter, namely the HR. ${ }^{11}$ We made use of: Soapy, ${ }^{44}$ a Monte Carlo AO simulation tool to replicate the atmospheric conditions encountered on-sky and the influence on the device's performance; and BeamProp by RSoft, ${ }^{45}$ which is a finite-difference beam propagation solver to model the light propagation through the components.

The configuration of the simulation was the following: first, Soapy was configured to produce an AO corrected star, and then the frames were used as an input for the BeamProp simulations.

\subsection{Soapy set-up}

The HR was tested on-sky with CANARY ${ }^{46}$ at the William Herschel Telescope. Therefore, Soapy was configured to approximate the sky conditions during the observation run (see Table 1). Soapy was used to simulate three different AO operation modes as on-sky with CANARY, namely closed-loop, open-loop and tip-tilt. In closedloop AO mode of operation CANARY provided correction at an update rate of $150 \mathrm{~Hz}$, both for tip-tilt and higher order aberrations, while for the tip-tilt AO mode of operation the integrator loop gain on the high-order modes was reduced to a small value of the order of 0.001, resulting in the correction of the PSF position in real-time, but not for the PSF shape which was provided without high-order AO correction. As for the case of open-loop mode of correction, the gain on the tip-tilt correction was further reduced in order to have the PSF remain in the reference location providing optimum coupling, but without having high-temporal frequency correction. Also, a value of $0.15 \mathrm{~m}$ for the seeing parameter (Fried parameter- $\mathrm{r}_{0}$ ) was selected, representative of the aforementioned on-sky experiments. ${ }^{11}$

Soapy produced 200 near infrared (NIR) data frames, each with a $6 \mathrm{~ms}$ exposure time as an input to BeamProp. Each Soapy frame covers a 3.0 arcseconds squared field on-sky, slightly more than three times the HR entrance angular size. In contrast to the data acquired on-sky, the Soapy frames provided both phase and amplitude information, which proved to be crucial for the simulation results (see Section 3.1).

\subsection{BeamProp set-up}

In the next stage, the frames from Soapy were used as an input for BeamProp simulations; the HR's angular size was set to 1.1 milliarcseconds. The design structure of the device was followed as described in Ref. 11. The HR is composed of a PL section followed by a ULI reformatter section, ending in a slit profile output.

To perform the simulations in BeamProp, the refractive index information is a crucial parameter, both for the core and the cladding materials. For the HR the initial PL section of multicore fiber was made by fused silica having step-index Ge-doped cores fabricated as in Ref. 30,47, and for the following reformatter section a 


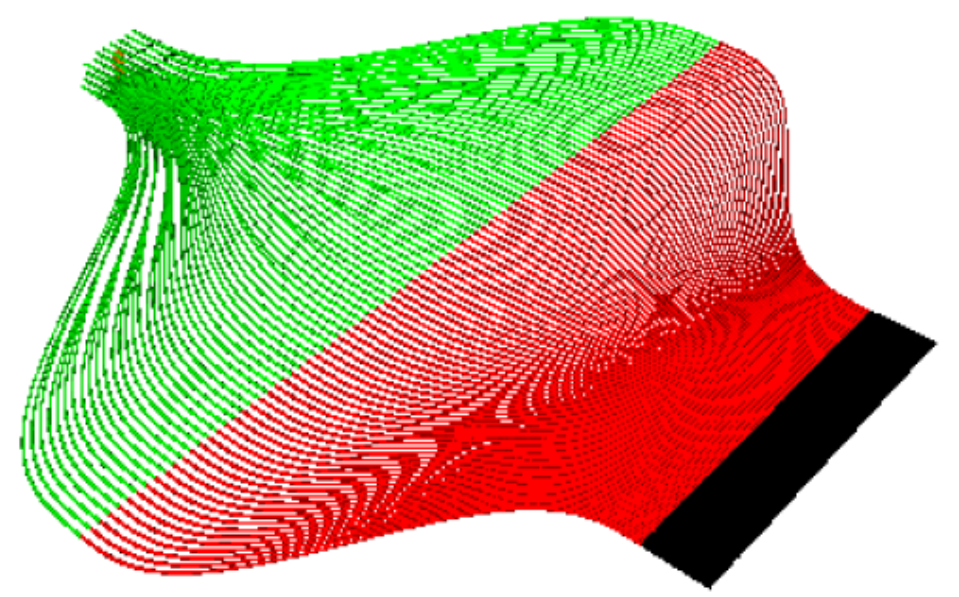

Figure 1. The hybrid reformatter 3D structure, without the initial photonic lantern section included in the plot, visualized in the RSoft's CAD environment. The colors show the 3 different transition planes used.

substrate of borosilicate glass was used (SCHOTT AF45) ${ }^{48}$ for the inscription of the waveguides with a $\mathrm{n}_{\mathrm{cl}}$ of $\sim 1.4974$ at $1550 \mathrm{~nm}$. As the are no refractive index measurements of the waveguide profiles for the device, the values from Ref. 32 were used $\left(\Delta=\frac{n_{\text {core }}-n_{\text {clad }}}{n_{\text {core }}} \approx 1.76 \times 10^{-3}\right)$ as they are considered to be close to our device, despite the small expected differences resulting from the inscription parameters (see Table 2) and the difference in glass. The 3D structure of the ULI section of the HR as shown in RSoft's CAD, is illustrated in Figure 1.

In general, in the BeamProp simulation tool the material propagation loss is a freely chosen parameter depending on the material and its properties. Therefore, in order to add greater precision in our simulations we ran tests using a propagation loss of $0.1 \mathrm{~dB} / \mathrm{cm}^{49}$ This value is an optimistic estimate representing relatively low losses when compared to the losses due to the geometric design $(<2 \%$ over the HR length). Further tests will be performed in future modeling with optimized devices.

Table 2. Comparison of ULI inscription parameters used in Ref. 32 and Ref. 11.

\begin{tabular}{lcc}
\hline Parameters & Thomson et al. & hybrid reformatter $(\mathrm{HR})$ \\
\hline$n_{\mathrm{cl}}(@ 1550 \mathrm{~nm})$ & $\sim 1.49$ & $\sim 1.4974$ \\
Pulse Energy $(\mathrm{nJ})$ & 165 & 174 \\
Pulse repetition rate $(\mathrm{kHz})$ & 500 & 500 \\
Pulse duration $(\mathrm{fs})$ & $350(1047 \mathrm{~nm})$ & $430(1030 \mathrm{~nm})$ \\
\hline
\end{tabular}

\subsection{Throughput measurement}

In order to calculate the total throughput $\left(T_{\text {tot }}\right)$ and transmission properties of the device from simulations the following formula was used:

$$
T_{\mathrm{tot}}=\frac{F_{\text {out }}(i)}{F_{\text {in }}(i)}, \quad i=\# \text { frames }
$$

where $F_{\text {out }}$ is the output of the slit and $F_{\text {in }}$ is the sum of the coupled flux of the input field.

\section{SIMULATION RESULTS}

\subsection{Output power performance results}

Here we present the simulation results. BeamProp simulations were performed using Soapy output data (amplitude and phase information). As the on-sky frames obtained with CANARY only record intensity these were 


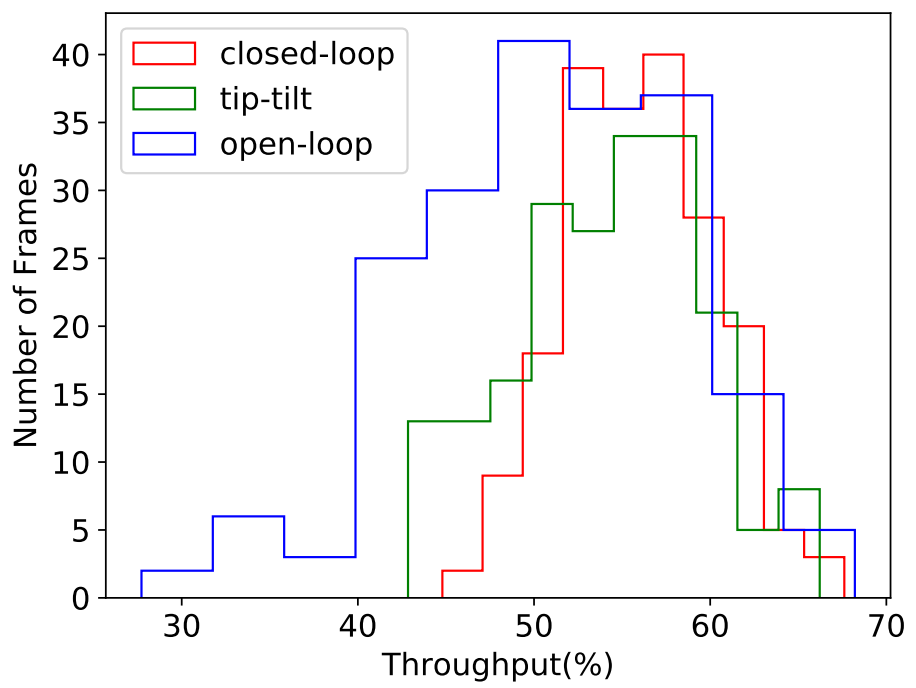

Figure 2. Histogram distribution of throughput measurements from simulations shown for three different AO modes. Red color represents the closed-loop AO mode of correction, green shows the tip-tilt correction and blue shows open-loop correction.

not used with BeamProp. This is due to the lack of phase information, which yields incorrect results (see Ref. 8).

Figure 2 shows the results of simulating 200 frames with Soapy. The throughput of the HR for closed-loop AO mode of operation was measured to be $56 \pm 4(\%)$. In tip-tilt operation mode the output was measured to be $55 \pm 5$ (\%); and for the open-loop correction results were $51 \pm 7(\%)$.

The ratio between the closed-loop and tip-tilt AO modes of correction plotted as a function of the device MM entrance input size for the Soapy averaged intensity data, is shown in Figure 3. This is calculated to better understand the coupling performance. The figure shows that the spatial size of the MM entrance of the device is larger than the spatial size of the input. Hence, all the light is coupled into the HR because of its large collecting area.

\subsection{Optimization results}

When the HR was designed and manufactured in 2014 there was no attempt to fully model it. Therefore, there are many areas for further optimizing the device. To study this, a Monte Carlo simulation run was performed to investigate the optimal length of transition positions in the ULI section of the device (see Figure 1).

A gradual transition of the ULI waveguides position in between the three different planes (see Figure 1) is helpful to ensure low loss of light. ${ }^{30}$ Nevertheless, there is a trade off between material and bend losses against the device's length, when making use of the ULI technique to inscribe waveguides in materials.

In order to optimize further the throughput performance of the HR, a Gaussian input (close to the diffraction limit) with a $25 \mu \mathrm{m}$ mode field diameter (MFD) generated by RSoft was chosen. Injecting this input, we performed a short Monte Carlo simulation on the HR, in order to optimize each of its reformatter section transition planes for transmission by scanning for a variety of different lengths between the 3 transition planes of the reformatter section. Preliminary results show an improvement in theoretical transmission by $2 \%$ (a $6 \%$ improvement in performance), leading to a shorter more compact device. However, transition planes can be further improved to be lower in loss (more adiabatic). 


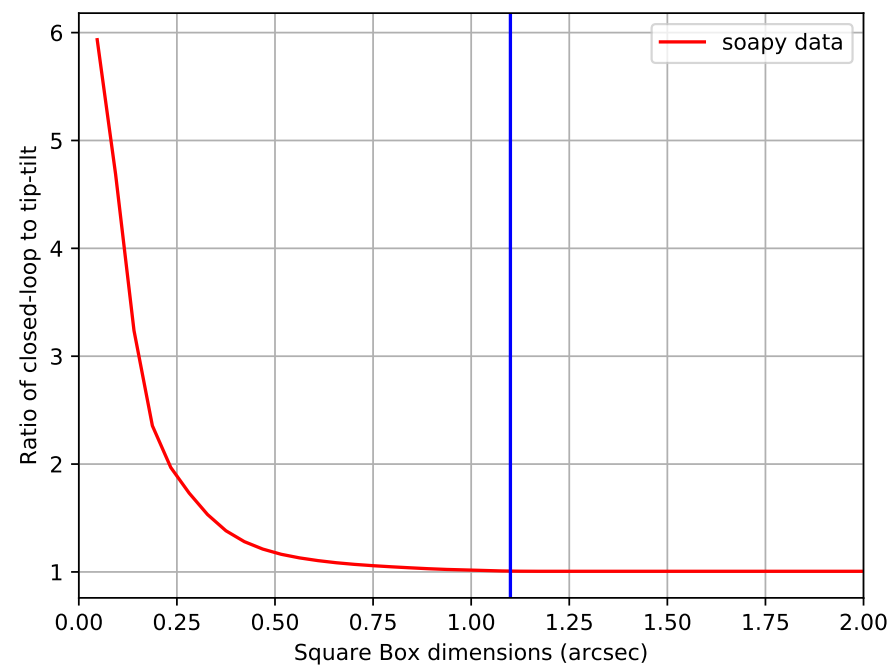

Figure 3. The behavior of closed-loop and tip-tilt AO mode ratios of EE related to the spatial scale (circular radius centered), plotted for simulated (Soapy) averaged (intensity) data. The vertical blue line represents the circular entrance size of the PL section of the HR.

\subsection{Modal noise results}

Aiming to investigate the modal noise properties of our theoretical HR device, we followed a similar procedure to that described by Ref. 8 . We choose the $1550 \mathrm{~nm}$ wavelength region to perform our simulations. We analyze the results from it by measuring the barycentric movement of the slit output ${ }^{50,51}$ examining its stability in the near field regime.

The dimension of the MFD and its barycentric movements were measured, from the simulation results. We also examined whether there are disturbances of the injected field to the device, which lead to different speckle distribution at the reformatter's slit output. Results of our analysis are presented in Figure 4. There the averaged (intensity) slit images are shown, as well as the calculated MFD size from fitting Gaussian distributions along the slit, and finally the barycentric movement of the MFD across the slit. The results of the barycentric movement of the slit, are expressed as a ratio of one-thousandth of the waveguide's core diameter size $(d / 1000)$. It is immediately apparent from the figure that the average semi-amplitude variation of the barycenter is of the order of $10^{-4}(d / 1000)$ which is small enough and meets our expectations for the modal noise properties of this device compared to other devices (Ref. 8), as discussed further in section 4.3. The aforementioned simulation results were obtained without taking into account any manufacturing errors in the slit straightness. As expected, the existence of such variations affect negatively the spectral resolving power of a spectrograph by causing noise and uncertainties in the measured spectra.

\section{DISCUSSION}

\subsection{Throughput performance}

Following the procedure as described in section 2 for our simulations, we obtained a throughput difference between the simulations and the on-sky output performance, of the order of 3 to 8 per-cent (see Table 3). These small differences in throughput results are due to the following reasons: first the on-sky AO raw data during the night of observation were not available at this point, therefore the AO performance per mode of correction could not be matched with precision; second the changing on-sky atmosphere conditions during the observation run; last, the assumption of perfect waveguide's structure and geometry in our simulations, without accounting for the manufacturing errors. 

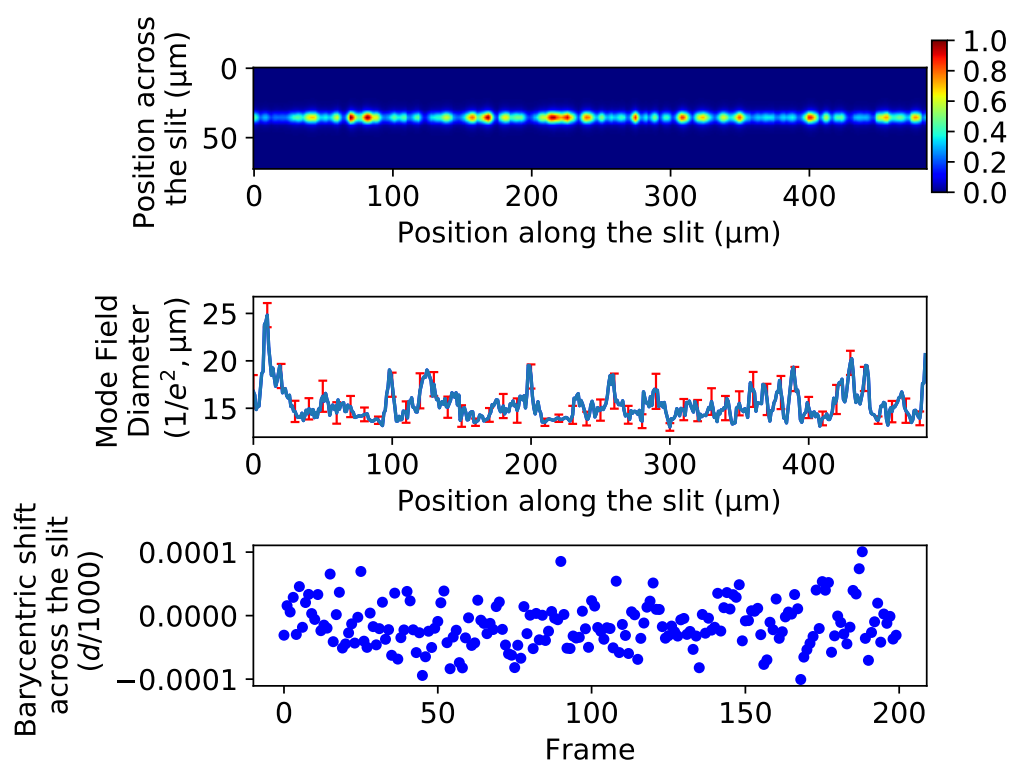

Figure 4. Top: Averaged (intensity) near-field images of the slit output from BeamProp simulations (@1550 nm). Middle: MFD profile size measurements of the slit with $1 \sigma$ errors from the individual images. Bottom: Barycentric variations across the slit of the device calculated from our dataset.

Table 3. Percentage results of throughput from simulations in comparison with the on-sky conditions.

\begin{tabular}{lcc}
\hline \multicolumn{3}{c}{ Data and results } \\
\hline \hline AO mode & On-sky & Soapy + BeamProp \\
\hline closed-loop (\%) & $53 \pm 4$ & $56 \pm 4$ \\
tip-tilt (\%) & $47 \pm 5$ & $55 \pm 5$ \\
open-loop (\%) & $48 \pm 5$ & $51 \pm 7$ \\
\hline
\end{tabular}

\subsection{Coupling of evanescent field}

The combination of a PL feeding a ULI reformatter creating the HR device resulted in the elimination of the undesired coupling of the evanescent field into the device, encountered in Ref. 8. This is due to the high refractive index difference among the cores and the cladding of the PL that guide light only inside the cores and not in the surrounding cladding.

\subsection{Modal noise}

It is apparent from the bottom part of Figure 4 that the barycenter movement of the slit is not completely stable, but has some variations of minor importance, even though the slit is designed to be completely straight. As a result, it will slightly affect the spectral resolving power of the spectrograph, but not to the same magnitude as when compared to a conventional fiber. ${ }^{51}$ To confirm our hypothesis, we performed two experiments; we applied the same method as in section 3.3 to a common state-of-the-art MM fiber with an octagonal cross section of 50 $\mu \mathrm{m}$ in diameter ${ }^{51}$ and a common SM fiber $8.2 \mu \mathrm{m}$ in diameter (Corning SMF-28 Optical Fiber).

It is apparent from results that the HR modal noise properties are similar to the SM fiber, while their barycentric movements are three orders of magnitude less than the MM octagonal fiber results (see Figure 5). Our results are quantitatively similar to other related results in the literature (e.g. Ref. 52).

\section{CONCLUSIONS}

We have performed a simulation experiment regarding the performance of an existing astrophotonic spatial reformatter, the hybrid reformatter. We used Soapy, ${ }^{44}$ a simulation program to replicate the on-sky conditions and its influence on the device's performance, and BeamProp by RSoft, ${ }^{45}$ a finite-difference beam propagation solver to model the light propagation through out device using data produced by Soapy. 

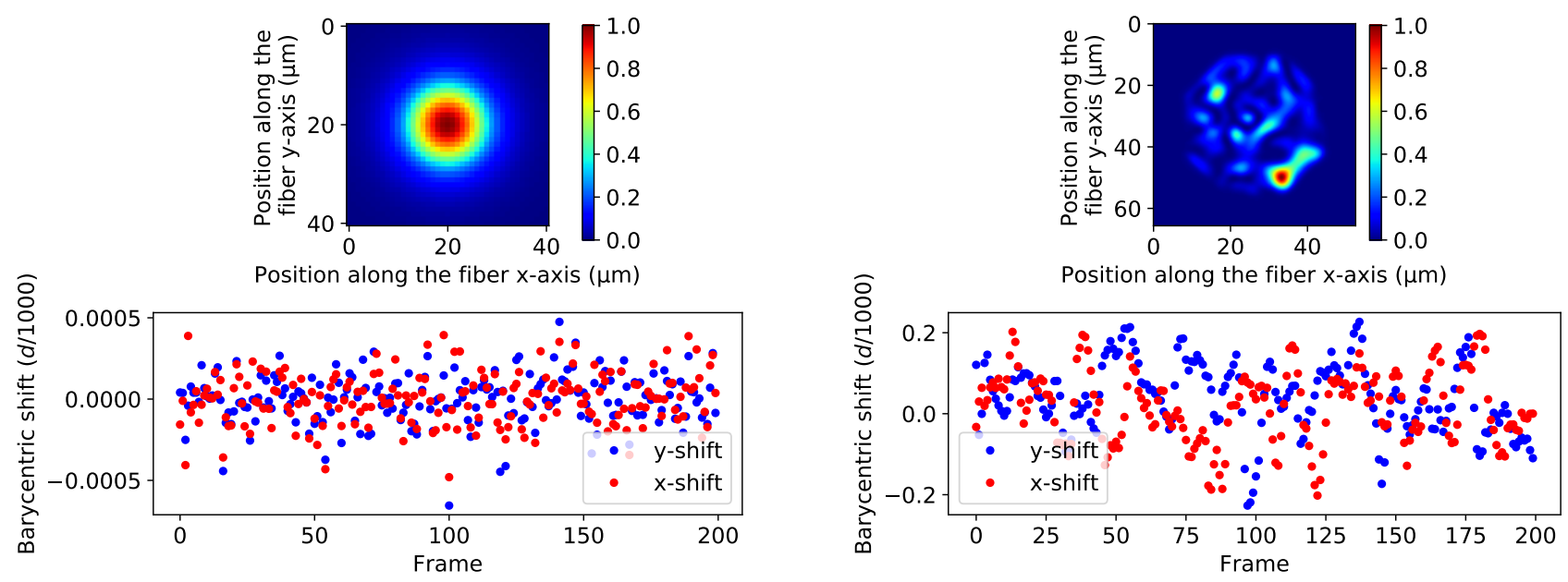

Figure 5. Left sub-figure: Top: Common near-field image (intensity) of the $8.2 \mu \mathrm{m}$ SM fiber output from BeamProp simulations. Bottom: Barycentric movement measurements along the fiber x-axis (red) and y-axis (blue) derived from individual images. Right sub-figure: Top: Common near-field image (intensity) of the $50 \mu \mathrm{m}$ octagonal fiber output from BeamProp simulations. Bottom: Barycentric movement measurements along the fiber x-axis (red) and y-axis (blue) derived from individual images.

Our simulation results were close to the on-sky reported values, yielding a device performance of $56 \pm 4 \%$ in closed-loop (compared to $53 \pm 4 \%$ for on-sky), $55 \pm 5 \%$ in tip-tilt (compared to $47 \pm 5 \%$ for on-sky) and 51 $\pm 7 \%$ in open-loop (compared to $48 \pm 5 \%$ for on-sky). The variable atmospheric seeing conditions encountered on-sky during the night of observations, which were not taken into account in this simulation, explain these differences in throughput.

Furthermore, we investigated the modal noise properties of the hybrid reformatter. Our results show that although the modal noise is not entirely absent, it is improved by three orders of magnitude compared to a typical MM fiber with an octagonal cross section.

Additional simulations were performed to optimize the device's transmission performance, resulting in a $2 \%$ improvement in theoretical transmission ( $\sim 6 \%$ improvement in performance) over the model of the original device. Simulating the performance of components including atmospheric effects proves to be crucial for improving their characteristics.

Our method as well as its outcome, shows that performing similar simulations to enhance the designs of future astronomical components is essential. Increasing their measurement precision while allowing for better calibration, and finally making them more compact in size and be compatible to the new generation of Extremely Large Telescopess (ELTs).

Future plans include the further optimization of the hybrid reformatter ${ }^{11}$ with a high potential to manufacture the optimized version.

\section{Acknowledgements}

This work was supported by the Deutsche Forschungsgemeinschaft (DFG) through project 326946494, 'Novel Astronomical Instrumentation through photonic Reformatting'. Robert J. Harris is funded/supported by the Carl-Zeiss-Foundation. R.R.T sincerely thanks the UK Science and Technology Facilities Council (STFC) for support through an STFC Consortium Grant (ST/N000625/1).

This research made use of Astropy, a community-developed core Python package for Astronomy (Astropy Collaboration, 2013), ${ }^{53}$ Numpy $^{54}$ and Matplotlib ${ }^{55}$ 


\section{REFERENCES}

[1] Mayor, M., Pepe, F., Queloz, D., Bouchy, F., Rupprecht, G., Lo Curto, G., Avila, G., Benz, W., Bertaux, J.-L., Bonfils, X., Dall, T., Dekker, H., Delabre, B., Eckert, W., Fleury, M., Gilliotte, A., Gojak, D., Guzman, J. C., Kohler, D., Lizon, J.-L., Longinotti, A., Lovis, C., Megevand, D., Pasquini, L., Reyes, J., Sivan, J.-P., Sosnowska, D., Soto, R., Udry, S., van Kesteren, A., Weber, L., and Weilenmann, U., "Setting New Standards with HARPS," The Messenger 114, 20-24 (Dec. 2003).

[2] Quirrenbach, A., Amado, P. J., Caballero, J. A., Mundt, R., Reiners, A., Ribas, I., Seifert, W., Abril, M., Aceituno, J., Alonso-Floriano, F. J., Anwand-Heerwart, H., Azzaro, M., Bauer, F., Barrado, D., Becerril, S., Bejar, V. J. S., Benitez, D., Berdinas, Z. M., Brinkmöller, M., Cardenas, M. C., Casal, E., Claret, A., Colomé, J., Cortes-Contreras, M., Czesla, S., Doellinger, M., Dreizler, S., Feiz, C., Fernandez, M., Ferro, I. M., Fuhrmeister, B., Galadi, D., Gallardo, I., Gálvez-Ortiz, M. C., Garcia-Piquer, A., Garrido, R., Gesa, L., Gómez Galera, V., González Hernández, J. I., Gonzalez Peinado, R., Grözinger, U., Guàrdia, J., Guenther, E. W., de Guindos, E., Hagen, H.-J., Hatzes, A. P., Hauschildt, P. H., Helmling, J., Henning, T., Hermann, D., Hernández Arabi, R., Hernández Castaño, L., Hernández Hernando, F., Herrero, E., Huber, A., Huber, K. F., Huke, P., Jeffers, S. V., de Juan, E., Kaminski, A., Kehr, M., Kim, M., Klein, R., Klüter, J., Kürster, M., Lafarga, M., Lara, L. M., Lamert, A., Laun, W., Launhardt, R., Lemke, U., Lenzen, R., Llamas, M., Lopez del Fresno, M., López-Puertas, M., López-Santiago, J., Lopez Salas, J. F., Magan Madinabeitia, H., Mall, U., Mandel, H., Mancini, L., Marin Molina, J. A., Maroto Fernández, D., Martín, E. L., Martín-Ruiz, S., Marvin, C., Mathar, R. J., Mirabet, E., Montes, D., Morales, J. C., Morales Muñoz, R., Nagel, E., Naranjo, V., Nowak, G., Palle, E., Panduro, J., Passegger, V. M., Pavlov, A., Pedraz, S., Perez, E., Pérez-Medialdea, D., Perger, M., Pluto, M., Ramón, A., Rebolo, R., Redondo, P., Reffert, S., Reinhart, S., Rhode, P., Rix, H.-W., Rodler, F., Rodríguez, E., Rodríguez López, C., Rohloff, R. R., Rosich, A., Sanchez Carrasco, M. A., Sanz-Forcada, J., Sarkis, P., Sarmiento, L. F., Schäfer, S., Schiller, J., Schmidt, C., Schmitt, J. H. M. M., Schöfer, P., Schweitzer, A., Shulyak, D., Solano, E., Stahl, O., Storz, C., Tabernero, H. M., Tala, M., Tal-Or, L., Ulbrich, R.-G., Veredas, G., Vico Linares, J. I., Vilardell, F., Wagner, K., Winkler, J., Zapatero Osorio, M.-R., Zechmeister, M., Ammler-von Eiff, M., Anglada-Escudé, G., del Burgo, C., Garcia-Vargas, M. L., Klutsch, A., Lizon, J.-L., Lopez-Morales, M., Ofir, A., Pérez-Calpena, A., Perryman, M. A. C., Sánchez-Blanco, E., Strachan, J. B. P., Stürmer, J., Suárez, J. C., Trifonov, T., Tulloch, S. M., and Xu, W., "CARMENES: an overview six months after first light," Proc. SPIE 9908, 990812 (Aug. 2016).

[3] Coudé du Foresto, V., "Integrated optics in astronomical interferometry [invited]," IAU Symposium 158, 261 (1994).

[4] Crepp, J. R., "Improving planet-finding spectrometers," Science 346(6211), 809-810 (2014).

[5] Schwab, C., Leon-Saval, S. G., Betters, C. H., Bland-Hawthorn, J., and Mahadevan, S., "Single Mode, Extreme Precision Doppler Spectrographs," IAU Symposium 293, 403-406 (Apr. 2014).

[6] Jovanovic, N., Schwab, C., Cvetojevic, N., Guyon, O., and Martinache, F., "Enhancing Stellar Spectroscopy with Extreme Adaptive Optics and Photonics," PASP 128, 121001 (Dec. 2016).

[7] Bland-Hawthorn, J. and Horton, A., "Instruments without optics: an integrated photonic spectrograph," Proc. SPIE 6269, 62690N (jun 2006).

[8] Anagnos, T., Harris, R. J., Corrigan, M. K., Reeves, A. P., Townson, M. J., MacLachlan, D. G., Thomson, R. R., Morris, T. J., Schwab, C., and Quirrenbach, A., "Simulation and Optimization of an Astrophotonic Reformatter," ArXiv e-prints (May 2018).

[9] Harris, R. J. and Allington-Smith, J. R., "Applications of Integrated Photonic Spectrographs in astronomy," MNRAS 428, 3139-3150 (Feb. 2013).

[10] Spaleniak, I., Jovanovic, N., Gross, S., Ireland, M. J., Lawrence, J. S., and Withford, M. J., "Integrated photonic building blocks for next-generation astronomical instrumentation II: the multimode to single mode transition," Optics Express 21, 27197 (Nov. 2013).

[11] MacLachlan, D. G., Harris, R. J., Gris-Sánchez, I., Morris, T. J., Choudhury, D., Gendron, E., Basden, A. G., Spaleniak, I., Arriola, A., Birks, T. A., Allington-Smith, J. R., and Thomson, R. R., "Efficient photonic reformatting of celestial light for diffraction-limited spectroscopy," MNRAS 464, 4950-4957 (Feb. 2017). 
[12] Vogt, S. S., Allen, S. L., Bigelow, B. C., Bresee, L., Brown, B., Cantrall, T., Conrad, A., Couture, M., Delaney, C., Epps, H. W., Hilyard, D., Hilyard, D. F., Horn, E., Jern, N., Kanto, D., Keane, M. J., Kibrick, R. I., Lewis, J. W., Osborne, J., Pardeilhan, G. H., Pfister, T., Ricketts, T., Robinson, L. B., Stover, R. J., Tucker, D., Ward, J., and Wei, M. Z., "HIRES: the high-resolution echelle spectrometer on the Keck 10-m Telescope," Proc. SPIE 2198, 362 (June 1994).

[13] Noguchi, K., Aoki, W., Kawanomoto, S., Ando, H., Honda, S., Izumiura, H., Kambe, E., Okita, K., Sadakane, K., Sato, B., Tajitsu, A., Takada-Hidai, M., Tanaka, W., Watanabe, E., and Yoshida, M., "High dispersion spectrograph (hds) for the subaru telescope," Publications of the Astronomical Society of Japan 54(6), 855-864 (2002).

[14] Tollestrup, E. V., Pazder, J., Barrick, G., Martioli, E., Schiavon, R., Anthony, A., Halman, M., and Veillet, C., "GRACES, the Gemini remote access CFHT ESPaDOnS spectrograph: initial design and testing," Proc. SPIE 8446, 84462A (Sept. 2012).

[15] Cunningham, C., "Future optical technologies for telescopes," Nature Photonics 3, 239-241 (May 2009).

[16] Mueller, M., Baldwin, D., Bean, J., Bergner, H., Bigelow, B., Chun, M.-Y., Crane, J., Foster, J., Fżrész, G., Gauron, T., Guzman, D., Hertz, E., Jordán, A., Kim, K.-M., McCracken, K., Norton, T., Ordway, M., Park, C., Park, S., Podgorski, W. A., Szentgyorgyi, A., Uomoto, A., and Yuk, I.-S., "The opto-mechanical design of the GMT-consortium large earth finder (G-CLEF)," Proc. SPIE 9147, 91479A (Aug. 2014).

[17] Zerbi, F. M., Bouchy, F., Fynbo, J., Maiolino, R., Piskunov, N., Rebolo Lopez, R., Santos, N., Strassmeier, K., Udry, S., Vanzi, L., Riva, M., Basden, A., Boisse, I., Bonfils, X., Buscher, D., Cabral, A., Dimarcantonio, P., Di Varano, I., Henry, D., Monteiro, M., Morris, T., Murray, G., Oliva, E., Parry, I., Pepe, F., Quirrenbach, A., Rasilla, J. L., Rees, P., Stempels, E., Valenziano, L., Wells, M., Wildi, F., Origlia, L., Allende Prieto, C., Chiavassa, A., Cristiani, S., Figueira, P., Gustafsson, B., Hatzes, A., Haehnelt, M., Heng, K., Israelian, G., Kochukhov, O., Lovis, C., Marconi, A., Martins, C. J. A. P., Noterdaeme, P., Petitjean, P., Puzia, T., Queloz, D., Reiners, A., and Zoccali, M., "HIRES: the high resolution spectrograph for the E-ELT," Proc. SPIE 9147, 914723 (Aug. 2014).

[18] Dekany, R., Roberts, J., Burruss, R., Bouchez, A., Truong, T., Baranec, C., Guiwits, S., Hale, D., Angione, J., Trinh, T., Zolkower, J., Shelton, J. C., Palmer, D., Henning, J., Croner, E., Troy, M., McKenna, D., Tesch, J., Hildebrandt, S., and Milburn, J., "PALM-3000: Exoplanet Adaptive Optics for the 5 m Hale Telescope," ApJ 776, 130 (Oct. 2013).

[19] Agapito, G., Arcidiacono, C., Quirós-Pacheco, F., and Esposito, S., "Adaptive optics at short wavelengths. Expected performance and sky coverage of the FLAO system going toward visible wavelengths," Experimental Astronomy 37, 503-523 (Nov. 2014).

[20] Macintosh, B., Graham, J. R., Ingraham, P., Konopacky, Q., Marois, C., Perrin, M., Poyneer, L., Bauman, B., Barman, T., Burrows, A. S., Cardwell, A., Chilcote, J., De Rosa, R. J., Dillon, D., Doyon, R., Dunn, J., Erikson, D., Fitzgerald, M. P., Gavel, D., Goodsell, S., Hartung, M., Hibon, P., Kalas, P., Larkin, J., Maire, J., Marchis, F., Marley, M. S., McBride, J., Millar-Blanchaer, M., Morzinski, K., Norton, A., Oppenheimer, B. R., Palmer, D., Patience, J., Pueyo, L., Rantakyro, F., Sadakuni, N., Saddlemyer, L., Savransky, D., Serio, A., Soummer, R., Sivaramakrishnan, A., Song, I., Thomas, S., Wallace, J. K., Wiktorowicz, S., and Wolff, S., "First light of the Gemini Planet Imager," Proceedings of the National Academy of Science 111, 12661-12666 (Sept. 2014).

[21] Jovanovic, N., Martinache, F., Guyon, O., Clergeon, C., Singh, G., Kudo, T., Garrel, V., Newman, K., Doughty, D., Lozi, J., Males, J., Minowa, Y., Hayano, Y., Takato, N., Morino, J., Kuhn, J., Serabyn, E., Norris, B., Tuthill, P., Schworer, G., Stewart, P., Close, L., Huby, E., Perrin, G., Lacour, S., Gauchet, L., Vievard, S., Murakami, N., Oshiyama, F., Baba, N., Matsuo, T., Nishikawa, J., Tamura, M., Lai, O., Marchis, F., Duchene, G., Kotani, T., and Woillez, J., "The Subaru Coronagraphic Extreme Adaptive Optics System: Enabling High-Contrast Imaging on Solar-System Scales," PASP 127, 890 (Sept. 2015).

[22] Weitzel, L., Krabbe, A., Kroker, H., Thatte, N., Tacconi-Garman, L. E., Cameron, M., and Genzel, R., "3D: The next generation near-infrared imaging spectrometer.," $A \mathscr{E} A S$ 119, 531-546 (Nov. 1996).

[23] Allington-Smith, J., Murray, G., Content, R., Dodsworth, G., Davies, R., Miller, B. W., Jorgensen, I., Hook, I., Crampton, D., and Murowinski, R., "Integral Field Spectroscopy with the Gemini Multiobject Spectrograph. I. Design, Construction, and Testing," PASP 114, 892-912 (Aug. 2002). 
[24] Hook, I. M., Jørgensen, I., Allington-Smith, J. R., Davies, R. L., Metcalfe, N., Murowinski, R. G., and Crampton, D., "The Gemini-North Multi-Object Spectrograph: Performance in Imaging, Long-Slit, and Multi-Object Spectroscopic Modes," PASP 116, 425-440 (May 2004).

[25] Bland-Hawthorn, J., Lawrence, J., Robertson, G., Campbell, S., Pope, B., Betters, C., Leon-Saval, S., Birks, T., Haynes, R., Cvetojevic, N., and Jovanovic, N., "PIMMS: photonic integrated multimode microspectrograph," Proc. SPIE 7735, 77350N (July 2010).

[26] Harris, R. J., MacLachlan, D. G., Choudhury, D., Morris, T. J., Gendron, E., Basden, A. G., Brown, G., Allington-Smith, J. R., and Thomson, R. R., "Photonic spatial reformatting of stellar light for diffractionlimited spectroscopy," MNRAS 450(1), 428 (2015).

[27] Leon-Saval, S. G., Betters, C. H., and Bland-Hawthorn, J., "The Photonic TIGER: a multicore fiber-fed spectrograph," Proc. SPIE 8450, 84501K (Sept. 2012).

[28] Leon-Saval, S. G., Birks, T. A., Bland-Hawthorn, J., and Englund, M., "Single-mode performance in multimode fibre devices," in [Optical Fiber Communication Conference and Exposition and The National Fiber Optic Engineers Conference], Optical Fiber Communication Conference and Exposition and The National Fiber Optic Engineers Conference, PDP25, Optical Society of America (2005).

[29] Leon-Saval, S. G., Argyros, A., and Bland-Hawthorn, J., "Photonic lanterns," Nanophotonics 2, 429-440 (Dec. 2013).

[30] Birks, T. A., Gris-Sánchez, I., Yerolatsitis, S., Leon-Saval, S. G., and Thomson, R. R., "The photonic lantern," Adv. Opt. Photon. 7, 107-167 (Jun 2015).

[31] Yerolatsitis, S., Harrington, K., and Birks, T. A., "All-fibre pseudo-slit reformatters," Optics Express 25, 18713 (Aug. 2017).

[32] Thomson, R., Birks, T., Leon-Saval, S., Kar, A., and Bland-Hawthorn, J., "Ultrafast laser inscription of an integrated photonic lantern," Optics Express 19, 5698-5705 (Mar. 2011).

[33] Cvetojevic, N., Lawrence, J. S., Ellis, S. C., Bland-Hawthorn, J., Haynes, R., and Horton, A., "Characterization and on-sky demonstration of an integrated photonic spectrograph for astronomy," Opt. Express 17, 18643-18650 (Oct 2009).

[34] Cvetojevic, N., Jovanovic, N., Lawrence, J., Withford, M., and Bland-Hawthorn, J., "Developing arrayed waveguide grating spectrographs for multi-object astronomical spectroscopy," Opt. Express 20, 2062-2072 (Jan 2012).

[35] Probst, R. A., Wang, L., Doerr, H.-P., Steinmetz, T., Kentischer, T. J., Zhao, G., Hänsch, T. W., Udem, T., Holzwarth, R., and Schmidt, W., "Comb-calibrated solar spectroscopy through a multiplexed single-mode fiber channel," New Journal of Physics 17, 023048 (Feb. 2015).

[36] Lemke, U., Corbett, J., Allington-Smith, J., and Murray, G., "Modal noise prediction in fibre spectroscopy i. visibility and the coherent model," MNRAS 417(1), 689 (2011).

[37] Perruchot, S., Bouchy, F., Chazelas, B., Díaz, R. F., Hébrard, G., Arnaud, K., Arnold, L., Avila, G., Delfosse, X., Boisse, I., Moreaux, G., Pepe, F., Richaud, Y., Santerne, A., Sottile, R., and Tézier, D., "Higher-precision radial velocity measurements with the SOPHIE spectrograph using octagonal-section fibers," Proc. SPIE 8151, 815115 (Oct. 2011).

[38] McCoy, K. S., Ramsey, L., Mahadevan, S., Halverson, S., and Redman, S. L., "Optical fiber modal noise in the 0.8 to 1.5 micron region and implications for near infrared precision radial velocity measurements," Proc. SPIE 8446, 84468J (Sept. 2012).

[39] Bouchy, F., Díaz, R. F., Hébrard, G., Arnold, L., Boisse, I., Delfosse, X., Perruchot, S., and Santerne, A., "SOPHIE+: First results of an octagonal-section fiber for high-precision radial velocity measurements," $A \& A$ 549, A49 (Jan. 2013).

[40] Iuzzolino, M., Tozzi, A., Sanna, N., Zangrilli, L., and Oliva, E., "Preliminary results on the characterization and performances of ZBLAN fiber for infrared spectrographs," Proc. SPIE 9147, 914766 (Aug. 2014).

[41] Halverson, S., Roy, A., Mahadevan, S., and Schwab, C., "'modal noise" in single-mode fibers: A cautionary note for high precision radial velocity instruments," ApJ 814(2), L22 (2015).

[42] Spaleniak, I., MacLachlan, D. G., Gris-Sánchez, I., Choudhury, D., Harris, R. J., Arriola, A., AllingtonSmith, J. R., Birks, T. A., and Thomson, R. R., "Modal noise characterisation of a hybrid reformatter," Proc. SPIE 9912, 991228 (July 2016). 
[43] Cvetojevic, N., Jovanovic, N., Gross, S., Norris, B., Spaleniak, I., Schwab, C., Withford, M. J., Ireland, M., Tuthill, P., Guyon, O., Martinache, F., and Lawrence, J. S., "Modal noise in an integrated photonic lantern fed diffraction-limited spectrograph," Opt. Express 25, 25546-25565 (Oct 2017).

[44] Reeves, A., "Soapy: an adaptive optics simulation written purely in Python for rapid concept development," Proc. SPIE 9909, 99097F (July 2016).

[45] Synopsys, "Rsoft photonic system design suite version 2017.03."

[46] Myers, R. M., Hubert, Z., Morris, T. J., Gendron, E., Dipper, N. A., Kellerer, A., Goodsell, S. J., Rousset, G., Younger, E., Marteaud, M., Basden, A. G., Chemla, F., Guzman, C. D., Fusco, T., Geng, D., Le Roux, B., Harrison, M. A., Longmore, A. J., Young, L. K., Vidal, F., and Greenaway, A. H., "Canary: the on-sky ngs/lgs moao demonstrator for eagle," Proc. SPIE 7015, 70150E-70150E-9 (2008).

[47] Birks, T. A., Mangan, B. J., Díez, A., Cruz, J. L., and Murphy, D. F., "“Photonic lantern" spectral filters in multi-core Fiber," Optics Express 20, 13996 (June 2012).

[48] Meany, T., Gross, S., Jovanovic, N., Arriola, A., Steel, M. J., and Withford, M. J., "Towards low-loss lightwave circuits for non-classical optics at 800 and 1,550 nm," Applied Physics A: Materials Science 8 Processing 114, 113-118 (Jan. 2014).

[49] Nasu, Y., Kohtoku, M., and Hibino, Y., "Low-loss waveguides written with a femtosecond laser for flexible interconnection in a planar light-wave circuit," Opt. Lett. 30, 723-725 (Apr 2005).

[50] Rawson, E. G., Goodman, J. W., and Norton, R. E., "Frequency dependence of modal noise in multimode optical fibers," J. Opt. Soc. Am. 70, 968-976 (Aug 1980).

[51] Chen, C.-H., Reynolds, R. O., and Kost, A., "Origin of spectral modal noise in fiber-coupled spectrographs," Appl. Opt. 45, 519-527 (Jan 2006).

[52] Feger, T., Brucalassi, A., Grupp, F. U., Lang-Bardl, F., Holzwarth, R., Hopp, U., and Bender, R., "A testbed for simultaneous measurement of fiber near and far-field for the evaluation of fiber scrambling properties," Proc. SPIE 8446, 844692 (Sept. 2012).

[53] The Astropy Collaboration, Price-Whelan, A. M., Sipőcz, B. M., Günther, H. M., Lim, P. L., Crawford, S. M., Conseil, S., Shupe, D. L., Craig, M. W., Dencheva, N., Ginsburg, A., VanderPlas, J. T., Bradley, L. D., Pérez-Suárez, D., de Val-Borro, M., Aldcroft, T. L., Cruz, K. L., Robitaille, T. P., Tollerud, E. J., Ardelean, C., Babej, T., Bachetti, M., Bakanov, A. V., Bamford, S. P., Barentsen, G., Barmby, P., Baumbach, A., Berry, K. L., Biscani, F., Boquien, M., Bostroem, K. A., Bouma, L. G., Brammer, G. B., Bray, E. M., Breytenbach, H., Buddelmeijer, H., Burke, D. J., Calderone, G., Cano Rodríguez, J. L., Cara, M., Cardoso, J. V. M., Cheedella, S., Copin, Y., Crichton, D., DÁvella, D., Deil, C., Depagne, É., Dietrich, J. P., Donath, A., Droettboom, M., Earl, N., Erben, T., Fabbro, S., Ferreira, L. A., Finethy, T., Fox, R. T., Garrison, L. H., Gibbons, S. L. J., Goldstein, D. A., Gommers, R., Greco, J. P., Greenfield, P., Groener, A. M., Grollier, F., Hagen, A., Hirst, P., Homeier, D., Horton, A. J., Hosseinzadeh, G., Hu, L., Hunkeler, J. S., Ivezić, Ž., Jain, A., Jenness, T., Kanarek, G., Kendrew, S., Kern, N. S., Kerzendorf, W. E., Khvalko, A., King, J., Kirkby, D., Kulkarni, A. M., Kumar, A., Lee, A., Lenz, D., Littlefair, S. P., Ma, Z., Macleod, D. M., Mastropietro, M., McCully, C., Montagnac, S., Morris, B. M., Mueller, M., Mumford, S. J., Muna, D., Murphy, N. A., Nelson, S., Nguyen, G. H., Ninan, J. P., Nöthe, M., Ogaz, S., Oh, S., Parejko, J. K., Parley, N., Pascual, S., Patil, R., Patil, A. A., Plunkett, A. L., Prochaska, J. X., Rastogi, T., Reddy Janga, V., Sabater, J., Sakurikar, P., Seifert, M., Sherbert, L. E., Sherwood-Taylor, H., Shih, A. Y., Sick, J., Silbiger, M. T., Singanamalla, S., Singer, L. P., Sladen, P. H., Sooley, K. A., Sornarajah, S., Streicher, O., Teuben, P., Thomas, S. W., Tremblay, G. R., Turner, J. E. H., Terrón, V., van Kerkwijk, M. H., de la Vega, A., Watkins, L. L., Weaver, B. A., Whitmore, J. B., Woillez, J., and Zabalza, V., "The Astropy Project: Building an inclusive, open-science project and status of the v2.0 core package," ArXiv e-prints (Jan. 2018).

[54] Van der walt, S., Colbert, S. C., and Gaël, V., "The NumPy array: a structure for efficient numerical computation," Computing in Science E Engineering 13, 22-30 (2011).

[55] Hunter, J. D., "Matplotlib: A 2d graphics environment," Computing In Science E Engineering 9(3), 90-95 (2007). 